\title{
REGISTRANDO A PRODUÇÃO DO TEXTO-ALVO COM O TRANSLOG ${ }^{1}$
}

\section{LOGGING TARGET TEXT PRODUCTION WITH TRANSLOG}

\author{
Arnt Lykke JAKOBSEN ${ }^{2}$ \\ Tradução de: Jonathan VIEIRA ${ }^{3}$
}

\begin{abstract}
Resumo: O Translog é um programa de computador que registra os acionamentos de teclas envolvidos na produção de uma tradução. Para fins de pesquisa, esse programa pode ser usado para ampliar a observação direta e suplementar os protocolos de verbalização do pensamento em voz alta (do inglês, think-aloud protocol, ou TAP). Com informações detalhadas em tempo real sobre o processo de digitação, podemos estudar todo o processo de edição que acontece durante a produção de uma tradução, a quantidade de tempo despendido em determinadas tarefas de tradução e a conexão entre o tempo de pausa e o processamento de informações. Este artigo resume as diversas funções do programa Translog e descreve como os dados são gravados e como podem ser representados e analisados. Por fim, defende-se a triangulação, ou seja, a combinação de metodologias como forma de aprimorar a metodologia de pesquisa em tradução.
\end{abstract}

Palavras-chave: Translog. Acionamento de teclas. Processo tradutório.

\section{Metodologia de pesquisa processual e o contexto do Translog ${ }^{4}$}

Existem duas formas principais de se estudar o processo pelo qual uma tradução se concretiza, quais sejam: a observação direta ou a introspecção.

\subsection{Observação direta}

A observação direta é a maneira mais óbvia de se estudar o processo tradutório. Podemos observar: tradutores trabalhando e como lidam com uma tarefa de tradução; como e quando recorrem ao material do texto-fonte; com que frequência conferem a compreensão do texto original recorrendo a dicionários, enciclopédias ou outras fontes de informação, como

\footnotetext{
${ }^{1}$ Agradecemos ao autor a gentileza de ceder os direitos para a publicação desta versão traduzida. Referência do artigo original: JAKOBSEN, Arnt Lykke. Logging target text production with Translog. In: HANSEN, G. (Ed.). Probing the process in translation: methods and results. Copenhagen: Samfundslitteratur, 1999. p. 9-20.

${ }^{2}$ Copenhagen Business School

${ }^{3}$ Professor e tradutor de alemão e inglês. Possui Bacharelado em Tradução pela Universidade Federal da Paraíba (2015). Atua como diretor-geral do Centro de Línguas Estrangeiras da Prefeitura Municipal de João Pessoa (CELEST). E-mail: jonathan.vieira.jvs@gmail.com. Tradução supervisionada pela Prof $^{\mathrm{a}} \operatorname{Dr}^{\mathrm{a}}$ Tânia Liparini Campos.

${ }^{4}$ A documentação do programa Translog está disponível em duas publicações, a saber:

JAKOBSEN, A. L.; SCHOU, L. Translog documentation: version 1.0. In: HANSEN, G. (Ed.). Probing the process in translation: methods and results. Copenhagen: Samfundslitteratur, 1999. p. 151-186. Appendix. JAKOBSEN, A. L. Logging time delay in translation. In: HANSEN, G. (Ed.) LSP texts and the process of translation, Copenhagen Working Press in LSP 1. Copenhagen: Copenhagen Business School, 1998. p. 73-101.
} 
colegas de profissão ou recursos da internet; quantas vezes interrompem o trabalho para ir à geladeira, conversar ao telefone ou pela internet, ou se envolver em outras atividades relacionadas, ou não, com a tarefa. Também podemos observar: como e quando o texto de chegada é escrito; com que velocidade e com que ritmo é produzido; quantas vezes um segmento é revisado ao longo do processo tradutório ou após a conclusão de uma primeira versão da tradução; e com que frequência e por meio de quantas interrupções no processo se faz uso de fontes de informação, especialmente de recursos bilíngues.

É possível, através da observação simples e direta, encontrar respostas provisórias a indagações sobre a tradução e, a partir dessas respostas, formular hipóteses preliminares, porém, geralmente, a observação precisa ser complementada por medidas adicionais. Podemse tomar notas para auxiliar a memória durante a análise subsequente dos dados; e podem-se adotar diversas técnicas e ferramentas para melhorar a precisão das observações. Por exemplo, pode-se contar o número de consultas ao dicionário ou de revisões textuais; pode-se utilizar o tempo do relógio para registrar a duração da produção textual; pode-se empregar um gravador de vídeo para possibilitar que novas observações sejam feitas tanto pelo pesquisador original quanto por outros. A função primária de todas essas técnicas e ferramentas é aumentar nossos poderes naturais de observação e de recordação dos fatos. O Translog deve ser visto nesse contexto: é uma ferramenta de pesquisa por meio da qual podemos aumentar o poder e a precisão da observação direta.

\subsection{Introspecção}

A outra forma principal de investigar o processo tradutório é a introspecção, geralmente por meio da análise de protocolos de verbalização do pensamento em voz alta (do inglês, think-aloud protocols, ou TAP), um método que se originou na psicologia cognitiva e na psicolinguística. Desde meados da década de 1980, esses protocolos têm sido o método mais utilizado para investigar a processo tradutório ${ }^{5}$. Em experimentos que utilizam TAPs, solicita-se aos participantes que verbalizem seus pensamentos enquanto traduzem. A verbalização é gravada em áudio e, posteriormente, transcrita, podendo conter informações adicionadas pelo pesquisador, como: os participantes de pesquisa; os materiais utilizados; o progresso na produção do texto-alvo; o tempo despendido; e o comportamento não verbal.

${ }^{5}$ Cf. ERICSSON, K. A.; SIMON, H. A. Protocol analysis: verbal reports as data. Cambridge, Massachusetts: MIT, 1984. Jääskeläinen (1999, p. 36-52) apresenta um panorama geral das pesquisas em tradução orientadas para o processo, focando a abordagem de protocolos de verbalização do pensamento em voz alta. 
Muitas vezes, também se grava uma entrevista retrospectiva imediatamente após a realização da tarefa. Os dados obtidos por meio dos protocolos de verbalização do pensamento em voz alta são de natureza qualitativa e requerem uma análise especial. O Translog também deve ser visto nesse contexto.

\subsection{Observação direta, análise de protocolos e o Translog}

Uma das motivações para o desenvolvimento do programa Translog foi a necessidade de se encontrar um respaldo quantitativo para as hipóteses sobre o processo tradutório aventadas apenas com base em dados qualitativos. Outra motivação foi a suposição de que, por meio do desenvolvimento de um método de coleta de dados quantitativos, poder-se-ia estabelecer uma espécie de diálogo entre as abordagens qualitativa e quantitativa que resultaria em um refinamento sinérgico de ambos os métodos e ajudaria a melhorar os métodos de análise de dados. Por fim, surgiu a ideia de que seria possível melhorar a nossa capacidade de validação ou falsificação dos resultados através da triangulação de dados qualitativos e quantitativos sobre o mesmo fenômeno. Em outras palavras, o objetivo era desenvolver uma ferramenta de observação mais precisa do processo de produção textual, bem como contribuir para o desenvolvimento metodológico da pesquisa em tradução.

\section{Registrando dados processuais com o Translog}

Quando um participante de pesquisa traduz usando o Translog, o texto-fonte aparece na metade superior da tela. $\mathrm{O}$ texto-fonte pode ser exibido de várias maneiras, a depender do objetivo da pesquisa; e também é possível disponibilizar um dicionário eletrônico. O textoalvo do tradutor é redigido na metade inferior da tela.

Os registros que o Translog faz do processo de produção de um texto são muito simples: ele registra qual tecla é pressionada e quando. Sempre que uma tecla for acionada, o Translog registra a hora do dia e armazena a informação. Em suma, o Translog cria um registro de cada tecla acionada durante a produção do texto-alvo, de todas as revisões realizadas, de todas as consultas feitas ao dicionário (eletrônico), de todos os erros de digitação e incorreções cometidos - e de quando tudo aconteceu. ${ }^{6}$

\footnotetext{
${ }^{6}$ Isso é feito com a precisão permitida pelo relógio do sistema DOS, ou seja, um dezoito avos de segundo, ou 55 milissegundos. Embora o Translog tenha sido desenvolvido de forma independente, não é o único programa de
} 


\section{Representação dos dados do Translog}

O Translog tem duas funções principais para o gerenciamento e para a representação dos dados dos arquivos de registro (.log): uma de visualização (View) e outra de reprodução (Replay).

A função View do Translog cria uma representação linear de todos os dados registrados. Mais especificamente, ela exibe não só os caracteres usados para criar o texto (letras, números, sinais de pontuação) - incluindo trechos de texto excluídos ou ensaiados e modificados -, mas também todos os movimentos do cursor, consultas ao dicionário eletrônico e intervalos de tempo. Com essa função, temos acesso a informações não somente sobre o texto final, mas também sobre revisões textuais, consultas a dicionários e pausas ao longo do processo.

Assim, é possível representar e, portanto, traçar todo o caminho que um participante percorre até chegar à versão final do texto-alvo. Em vez de vermos apenas o produto final, como "the weakest" no exemplo da Figura 1, podemos observar todas as camadas subjacentes e preliminares de produção textual e de tomada de decisão que contribuíram para a elaboração da versão final (e.g., "the wea", "the "the weakest", "the very weakest", "the very weakest").

As informações temporais registradas são representadas por asteriscos ou por indicações do tempo absoluto de pausa (cf. Figura 1). Pode-se atribuir qualquer valor entre $0,01 \mathrm{~s}$ a $99,00 \mathrm{~s}$ aos asteriscos. Assim, por meio do traçado das pausas, ou do intervalo de tempo, entre a digitação de palavras ou letras, obtém-se uma linha do tempo (timeline) do processo de digitação. Dependendo do valor atribuído à unidade de tempo (i.e., o valor do asterisco), uma representação terá um grau de detalhe, ou uma "resolução", diferente no que diz respeito à informação temporal (cf. Figura 1).

A primeira representação, na Figura 1, com um valor de unidade de tempo de 25 s, nos diz que o tradutor escreveu primeiro "wea", em seguida excluiu "wea" e, então, digitou "weakest". Porém, não nos diz quase nada sobre a linha do tempo da produção de "the weakest".

computador de sua espécie (cf. EKLUNDH; KOLLBERG, 1995; KOLLBERG, 1998). Outro programa do gênero, chamado TESTRANS, foi relatado por Natalia Podolskaya, da Universidade Linguística de Moscou, na conferência de Tradução e Cognição em Savonlinna, entre 2 e 3 de outubro de 1998. 
Figura 1 - Quatro diferentes representações de "the weakest" no Translog com valores de unidade de tempo (asterisco) de 25 segundos, 1 segundo, 0,20 segundo e 0,04 segundo, respectivamente.

1. Translog 25.00

the wea $\bullet 4$ weakest

2. Translog 1.00

the $[*: 21.10]$ wea 4 weakest*****•

3. Translog 0.20

the* $[*: 21.10] \mathrm{we}^{*} \mathrm{a} * * 4<[*: 19.00] \mathrm{w} * \mathrm{e}^{*} \mathrm{a} * \mathrm{ke}^{*} \mathrm{~s} * \mathrm{t}\left[{ }^{*}: 5.65\right]$

4. Translog 0.04

$\mathrm{t} * * * * \mathrm{~h} * * * \mathrm{e} * * * * * * * * * \bullet[*: 21.10] \mathrm{W} * * * \mathrm{e} * * * * * * \mathrm{a}[*: 0.49]$

$[*: 19.00] \mathrm{W} * * * * * \mathrm{e} * * * * * \mathrm{a} * * * * * \mathrm{k} * * \mathrm{e} * * * * * * * * \mathrm{~s} * * * * * * \mathrm{t}[*: 5.65]$

A segunda representação nos mostra que houve uma longa pausa $(21,10 \mathrm{~s})$ antes da digitação de "wea", que as três letras foram rapidamente excluídas e que, depois, houve outra pausa longa (19,00 s) antes da digitação de "weakest". Finalmente, depois da produção de "weakest", houve uma pausa de cerca de 5,00 s de duração. Essa representação ilustra a distribuição típica de pausas de processamento inicial e intermediário e pausas de monitoramento final.

A terceira representação nos permite investigar pausas dentro das palavras. $\mathrm{O}$ valor de tempo de cada asterisco aqui é de um quinto de segundo, o que parece ser aproximadamente a velocidade de digitação do participante em tela. Essa constatação parece ser confirmada pelo fato de que, na digitação de "weakest", houve uma pausa de cerca de um quinto de segundo entre todas as letras, exceto entre o " $k$ " e o " $e$ " (cf. " $w * e^{*} a * k e^{*} s *$ "). Surpreendentemente, não houve pausa que correspondesse aos limites dos morfemas.

Com a quarta representação, aproximamo-nos de um limite inferior. A precisão da informação temporal é maior do que aquela das representações anteriores, mas deve-se considerar se a perda de legibilidade é compensada pelas observações que podem ser feitas nesse nível de decomposição.

Os pressupostos subjacentes à gravação de informações temporais e às várias formas de representar essas informações são: (i) existe uma correlação entre pausas durante a produção textual e o respectivo processamento cognitivo envolvido nesse caso; e, mais especificamente, (ii) essa correlação pode ser observada em diferentes níveis. A fundamentação geral para esses pressupostos está na observação do ritmo cognitivo na 
produção não reprodutiva da linguagem (SCHILPEROORD, 1996, p. 5). Assume-se que o perfil temporal do processo de escrita ou de tradução reflete tanto a quantidade quanto o tipo de processamento cognitivo que ocorre durante esse processo.

O Translog mede intervalos de tempo com grande precisão e possibilita estudar correlações dos padrões de distribuição de pausas não apenas com os elementos macrotextuais (parágrafos, orações e palavras), mas também com elementos microtextuais, isto é, abaixo do nível da palavra. Para diversos fins de pesquisa, a precisão em nível microtextual não é essencial e pode até gerar um emaranhado confuso de dados que dificultam ou inviabilizam observações relevantes. Uma das principais vantagens do Translog está na facilidade e flexibilidade com que pode ser calibrado e na velocidade com que todos os dados temporais registrados podem ser recalculados a fim de produzir a representação - linear ou dinâmica mais relevante para determinado propósito de pesquisa.

A gravação precisa de informações temporais pelo Translog possibilita reproduzir o processo de digitação (usando a função de reprodução, Replay) quantas vezes forem necessárias e em diferentes velocidades, seja para inspeção e análise mais detalhadas ou como um mecanismo de recordação ao participante da pesquisa sobre o que ele estava pensando durante o processo de produção textual. Por esse método, é possível obter informações muito precisas e detalhadas sobre aquilo em que um participante estava pensando em determinado momento do processo tradutório sem ter que se recorrer à técnica de TAP.

\section{Os dados do Translog}

Objetividade, precisão e exaustividade são qualidades muito importantes em dados de pesquisa, o que pode ser conseguido com a ajuda de um computador, que registra os dados de forma confiável, com automatismo, precisão, amplitude e objetividade. A máquina é completamente imparcial e isenta. Algo que não é um problema de tradução é tão interessante ou desinteressante como algo que é.

Muitos dos participantes de pesquisa que, até agora, se voluntariaram para participar dos experimentos tradutórios com o Translog indicaram que se esqueceram de que estavam fazendo parte de um experimento e disseram que fazer uma tradução no Translog era muito semelhante a fazer uma tradução em outro ambiente. Também informaram que não haviam ignorado o fato de que sua produção textual estava sendo registrada. Parece, portanto, que os

experimentos executados com o Translog têm validade ecológica: é possível extrair dados 
importantes de forma não intrusiva ou não invasiva que simula a tradução na vida real. A interface Writelog (a parte do Translog usada para experimentos) é tão fácil de navegar que o pesquisador não precisa estar presente durante um experimento.

Os dados do Translog nos lembram, muito claramente, que eles são, na melhor das hipóteses, apenas sintomas de uma atividade mental subjacente. Eles têm de ser interpretados antes de serem compreendidos como evidências de processos mentais específicos. Os dados do Translog não têm o rico caráter sugestivo muitas vezes encontrado nos dados qualitativos obtidos pela técnica de TAP. Os dados do Translog não "falam” por si sós até que comecemos a teorizar sobre eles. As pausas são todas, de certa forma, apenas lacunas ou períodos de silêncio que temos de preencher para chegarmos a alguma compreensão. Dependemos do desenvolvimento de ideias de como a tradução se dá para podermos começar a extrair significado dos dados do Translog.

No final das contas, não se trata de algo diferente da forma como devemos abordar os dados qualitativos. O que o relato verbal faz, essencialmente, é criar um segundo texto, o texto do protocolo, o qual, assim como a representação do Translog, não é uma descrição direta dos processos mentais que procuramos acessar. Os protocolos verbais e as representações do Translog são, portanto, iguais nesse sentido. Nenhum dos dois faz sentido a não ser que sejam baseados em algumas ideias ou teorias de como a tradução acontece.

\section{Desenho experimental}

Os experimentos que utilizam o Translog podem ser configurados de diferentes formas, dependendo de sua finalidade. Geralmente, recomenda-se um processo de coleta de dados em três etapas.

A primeira etapa envolve testes preliminares. Como parte desse teste inicial, pode-se solicitar aos participantes da pesquisa que executem uma tarefa no Writelog, para assegurar que estejam familiarizados com os aspectos práticos e técnicos da configuração do experimento. Para o pesquisador, o teste preliminar fornece informações não apenas sobre o conhecimento prévio dos participantes, mas também sobre uma série de parâmetros de desempenho, como velocidade de digitação, habilidades de revisão e habilidades gerais de navegação pelo programa.

Na segunda etapa, os participantes executam, individualmente ou em pares, uma tarefa de produção textual. No caso, uma tarefa de tradução pode durar entre cerca de dez e 120 
minutos. Nessa etapa do experimento, o registro dos dados com o Writelog pode ser combinado com a técnica de TAP, com a gravação de áudio ou vídeo e com a observação direta. Além disso, pode-se permitir que os participantes tenham acesso a informações de fontes diferentes daquelas fornecidas pelo Writelog, como, por exemplo, de dicionários impressos.

A terceira etapa geralmente consiste em uma entrevista retrospectiva realizada com ou sem a função Replay do Translog. A retrospecção imediata reforçada pela observação da reprodução do próprio processo de produção textual geralmente resulta em dados valiosos. ${ }^{7}$

Assim, os dados estão geralmente relacionados a uma das três etapas do experimento. A depender da utilização do Translog de forma independente ou em combinação com outros métodos de obtenção de dados, o pesquisador terá acesso aos dados em pelo menos quatro formatos diferentes: dados do Translog, TAP escrito (transcrição de informações gravadas por meio de equipamento de áudio/vídeo), entrevista retrospectiva escrita (transcrição de informações registradas por meio de equipamentos de áudio/vídeo) e/ou registros de áudio ou vídeo do comportamento, tanto verbal quanto não verbal, do participante.

\section{Analisando os dados do Translog}

Quando estudados separadamente, os dados do Translog podem ser analisados quanto a questões como velocidade de produção textual, extensão da revisão textual e de consultas a dicionário, bem como problemas de tradução, que são facilmente identificados por meio da distribuição das pausas e da ocorrência de revisões textuais. Os dados também se prestam a todo tipo de análise quantitativa, como, por exemplo: a razão entre o número total de acionamentos de teclas e o número total de caracteres na versão final do texto-alvo; a razão (em termos de tempo ou acionamentos de teclas) entre a primeira versão do texto e a versão revisada; o tempo médio de pausa entre palavras ou unidades de tradução; e a velocidade mínima ou máxima de produção textual entre sentenças.

Os dados do Translog também podem ser estudados em combinação com dados do processo tradutório de outras fontes, como TAP, entrevistas, relatos retrospectivos imediatos ou gravações de vídeo. Nesse caso, a sincronização dos dados é crucial. A Figura 2 mostra uma forma de sincronizar e integrar os dados do Translog com dados de protocolos verbais (em dinamarquês). Delimitando desenhos experimentais que possibilitam a coleta de dados de

\footnotetext{
${ }^{7}$ As funções pause-replay e resume-replay foram adicionadas às primeiras versões do Translog por sugestão dos membros de um projeto sobre o processo tradutório desenvolvido na Copenhagen Business School (CBS).
} 
um mesmo evento de tradução por meio de diferentes fontes, bem como a sincronização e combinação desses dados, é possível obter uma imagem mais clara do processo tradutório.

Figura 2 - Os dados do Translog mostram o tempo acumulado na coluna da esquerda e a relação com os dados dos protocolos verbais

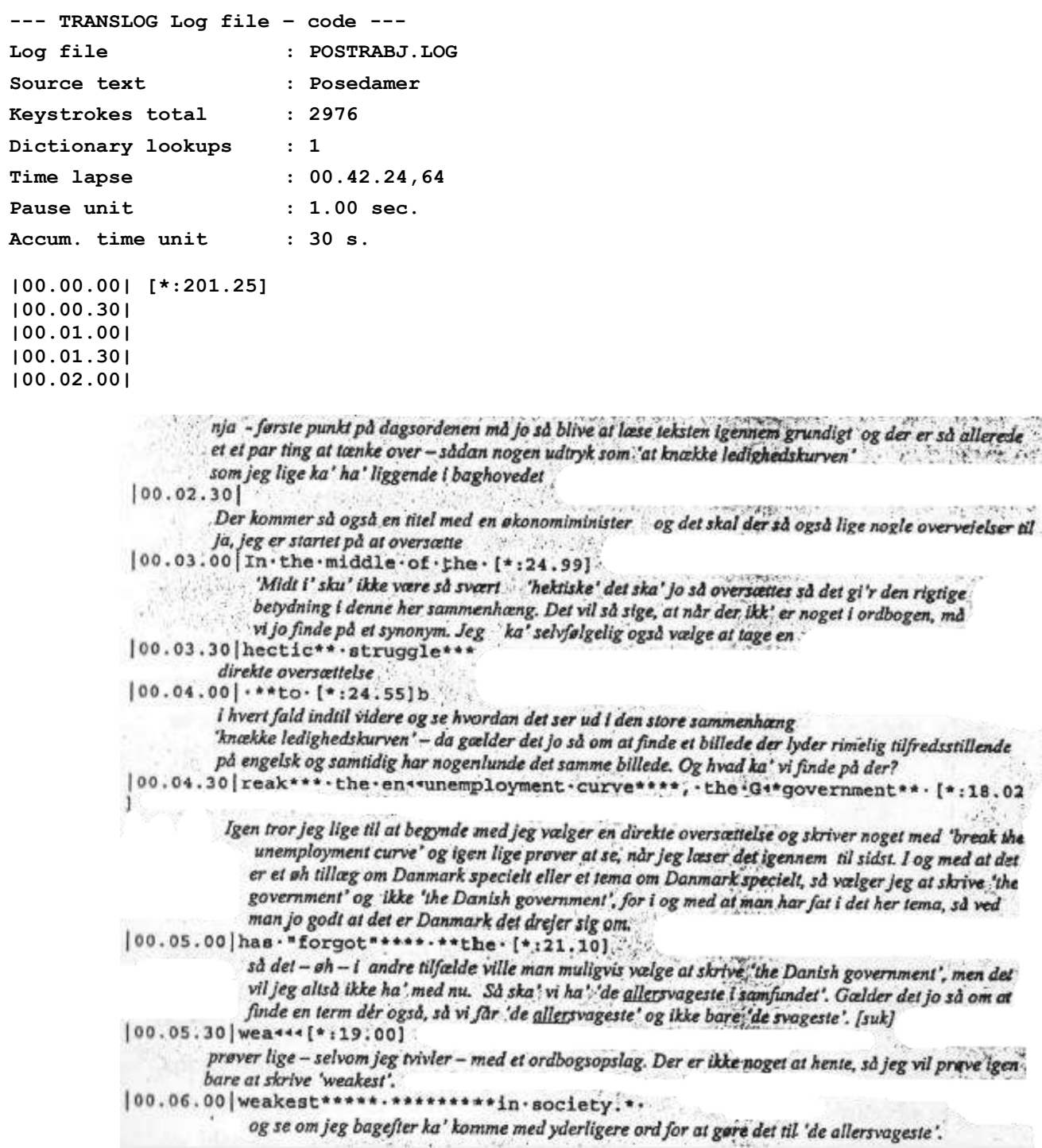

\section{Triangulação}

Nas ciências sociais, há uma longa tradição no emprego de métodos qualitativos e quantitativos de forma complementar. De acordo com Jick (1979), a maioria dos livros sobre pesquisa em ciências sociais, recomenda, desde os anos 1960, o uso de vários métodos, também conhecido como triangulação ou metodologia convergente (CAMPBELL; FISKE, 
1959; WEBB et al., 1966; SMITH, 1975; DENZIN, 1978, 1994; JICK, 1979). Como explicado por Smith (1975, p. 273), a metáfora da triangulação vem das estratégias militares e de navegação, que usam vários pontos de referência para localizar a posição exata de um objeto. Por analogia, por meio do estudo de dados - sobre um mesmo fenômeno ou objeto (no caso o processo tradutório) - coletados e interpretados a partir de diferentes métodos, nossas chances de conhecer esse objeto são maiores. Se a observação de fenômenos comparáveis permanece estável e convergente a partir da perspectiva de métodos diferentes, reduz-se a possibilidade de que sejam meros resultados dos artefatos metodológicos (cf. BOUCHARD, 1976, p. 268). A triangulação, que envolve a utilização de vários métodos diferentes, é, por conseguinte, essencialmente um meio de validação de dados de observação. Esse é o arquétipo das estratégias de triangulação.

Denzin (1978, p. 301) menciona a possibilidade de utilizar várias técnicas de coleta de dados como parte de um único método - é a triangulação dentro do método. Essa é outra possibilidade gerada pela combinação de dados quantitativos do Translog com dados qualitativos de protocolos verbais. Ao estudarmos o processo tradutório, podemos contrastar sistematicamente dados de protocolos verbais com dados coletados durante a retrospecção imediata, usar vários grupos de comparação, contrastar dados quantitativos de tempo de pausa e velocidade de digitação etc. Em tais casos, a triangulação é um meio de cruzar dados a fim de que haja consistência interna ou confiabilidade (JICK, 1979, p. 603).

A triangulação tem sido apontada como capaz não só de validar dados observacionais, mas também de capturar um retrato mais completo do objeto ou fenômeno em estudo. $\mathrm{O}$ uso de vários métodos pode "evidenciar algumas nuances particulares que, do contrário, podem ser negligenciadas por um método simples” (JICK, 1979, p. 603) e, assim, enriquecer a nossa compreensão do objeto.

\section{Conclusão}

Não há uma tradição consolidada de experimentos em condições laboratoriais e de trabalho com dados quantitativos no âmbito das ciências humanas na Europa. Muitos estudiosos da tradução, sem dúvida, apresentarão sérias reservas quanto ao uso de um programa como o Translog, porque sentem que estariam se comprometendo com um posicionamento behaviorista ou mesmo positivista. Eu gostaria de sugerir, no entanto, que a tradição de abordagens multimetodológicas das ciências sociais representa um desafio que devemos acolher. E um lugar óbvio para começar seria tentar identificar observações 
convergentes em dados qualitativos e quantitativos em relação a um mesmo evento de tradução. Ao triangular dados quantitativos e qualitativos, ao testar hipóteses com base no contraste entre dados qualitativos e dados quantitativos, e vice-versa, eu acredito que em breve conseguiremos aventar hipóteses mais robustas e mais bem embasadas sobre a tradução.

\section{Referências}

BOUCHARD, T. J. Unobtrusive measures: an inventory of uses. Sociological Methods and Research, v. 4, p. 267-300, 1976.

CAMPBELL, D. T.; FISKE, D. W. Convergent and discriminant validation by the multitraitmultimethod matrix. Psychological Bulletin, v. 56, p. 81-105, 1959.

DENZIN, N. K. The research act. 2. ed. Nova York: McGraw-Hill, 1978.

EKLUNDH, K. S.; KOLLBERG, P. Computer tools for tracing the writing process: from keystroke records to S-notation. In: RIJLAARSDAM, G.; COUZIJN, M.; VAN DEN

BERGH, H. (Ed.). Current research in writing: theories models and methodology. Amsterdã: Amsterdam University Press, 1995. p. 526-541.

JAKOBSEN, A. L. Logging time delay in translation. In: HANSEN, G. (Ed.). LSP texts and the process of translation, Copenhagen Working Papers ins LSP 1. Copenhagen: Copenhagen Business School, 1998a. p. 73-101.

JAKOBSEN, A. L. Understanding the process of translation: the contribution of time delay studies. Paper read at the ASLA symposium on Translation and Interpreting (Översättning och tolkning), Stockholm University, Estocolmo, 5-6 nov. 1998b.

JENSEN, A.; JAKOBSEN, A. L. Translating under time pressure: an empirical investigation of problem solving activity and translation strategies by non-professional and professional translators. Amsterdã: John Benjamins, 2000. p. 105-116.

JICK, T. D. Mixing qualitative and quantitative methods: triangulation in action.

Administrative Science Quarterly, v. 24, p. 602-611, 1979.

JÄÄSKELÄINEN, R. Tapping the process: an explorative study of the cognitive and affective factors involved in translating. Joensuu: Joensuun yliopisto, 1999.

KOLLBERG, P. S-notation - a computer based method for studying and representing text composition. Estocolmo: Stockholm University (Kungl. Tekniska Högskolan), 1998.

PODOLSKAYA, N. Translation process: a computer-aided modelling technique. Paper read at the conference on Translation and Cognition at Savonlinna School of Translation Studies, University of Joensuu, 2-3 out. 1998.

SCHILPEROORD, J. It's about time: temporal aspects of cognitive processes in text production. Amsterdã: Rodopi, 1996. 
SMITH, H. W. Strategies of social research: the methodological imagination. Englewood Cliffs, NJ: Prentice Hall, 1975.

WEBB, E. J.; CAMPBELL, D. T.; SCHWARTZ, R. D.; SECHREST, L. Unobtrusive measures: non-reactive research in the social sciences. Chicago: Rand McNally, 1966. 